\title{
La gestión de la equidad y la cooperación Tecnica para a reclasificación da oferta de interés turístico-cultural y la creación del cluster en Montevideo, Uruguay
}

Gestão do patrimônio e cooperação técnica na requalificação da oferta de interesse turístico-cultural e a criação do cluster em Montevidéu, Uruguai Heritage management and technical cooperation in the requalification of the touristic-cultural interest offer and the cluster creation in Montevideo, Uruguay

http://dx.doi.org/10.18472/cvt.17n1.2017.1053

Mary Sandra Guerra Ashton 〈marysga@feevale.br > Universidade Feevale (Feevale), Novo Hamburgo, RS, Brasil.

Edegar Luis Tomazzoni 〈eltomazzoni@usp.br > Universidade de São Paulo (USP), São Paulo, SP, Brasil.

Rossana Campodónico 〈campodon@gmail.com > Universidad de La República (Udelar), Montevideo, Uruguay.

CRONOLOGIA DO PROCESSO EDITORIAL

Recebimento do artigo: 25-jun-2015

Aceite: $16-$ dez-2016

FORMATO PARA CITAÇÃO DESTE ARTIGO

ASHTON, M. S. G.; TOMAZZONI, E. L.; CAMPODÓNICO, R. La gestión de la equidad y la cooperación Tecnica para a reclasificación da oferta de interés turístico-cultural y la creación del cluster en Montevideo, Uruguay. Caderno Virtual de Turismo. Rio de Janeiro, v. 17, n. 1, p. 58-72, abr. 2017.

REALIZAÇÃO

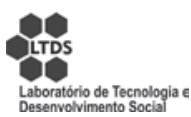

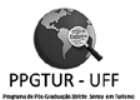

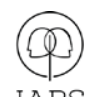

APOIO INSTITUCIONAL

EDIÇÃO

PATROCÍNIO

COPPE

UFR] 


\section{RESUMEN}

Este trabajo tiene como objetivo analizar el caso de Montevideo, en la gestión de sus activos y la formación del cluster de turismo cultural en 2008 para reclasificación de la oferta de interés turístico-cultural, desde la creación del conglomerado turístico, con con base en el marco teórico de la equidad y el turismo cultural, mirando a la cooperación potencial y técnica de las oportunidades de la capital uruguaia. Fue adoptado el método descriptivo y interpretativo y el estudio de caso con un enfoque cualitativo. Inicialmente, tratase de comprender la importancia de la gestión del patrimonio cultural de Montevideo para el turismo, contextualizada en las actividades de cooperación técnica de Montevideo. Entre los resultados, se espera obtener y proporcionar elementos conceptuales y metodológicos que podrían ser útiles para futuros análisis de la restauración de la oferta de cooperación cultural y técnica, poniéndolos a disposición de la comunidad académica y científica, para los gestores municipales y las empresas.

Palavras-chave: Turismo y Gestión. Cooperación Técnica. Patrimonio. Montevideo.

\section{ABSTRACT}

This work aims to analyze the Montevideo case in the management of its heritage and creation of the cultural tourism cluster in 2008, from the cultural assets offer requalification of observed touristic interest, since the tourism conglomerate creation. This analysis is based on the theoretical framework addressing heritage and cultural tourism, contemplating the potentialities and opportunities of the Uruguayan capital technical cooperation. It was adopted the descriptive-interpretative method and a case study with qualitative approach. Initially, it was aimed to understand the importance of Montevideo's cultural heritage management for tourism, contextualized in its technical cooperation actions. Among the results, it is expected to obtain and make available conceptual and methodological elements that may be useful for future analyzes that involve the requalification of the cultural assets offer and technical cooperation, making them available to the academicscientific community, as well as to municipal and business managers.

Keywords: Tourism and Management. Technical Cooperation. Heritage. Montevideo.

\section{RESUMO}

Este trabalho tem como objetivo principal analisar o caso de Montevidéu na gestão do seu patrimônio e formação do cluster de turismo cultural, em 2008, a partir da requalificação da oferta de bens culturais de interesse turístico, observado com a criação do conglomerado de turismo, com base no referencial teórico de patrimônio e turismo cultural, contemplando as potencialidades e as oportunidades de cooperação técnica da capital uruguaia. Adotou-se o método descritivo-interpretativo e o estudo de caso com abordagem qualitativa. Inicialmente, buscou-se compreender a importância da gestão do patrimônio cultural da capital para o turismo, contextualizado nas ações de cooperação técnica de Montevidéu. Entre os resultados, obtiveram-se elementos conceituais e metodológicos que possam ser úteis para futuras análises que envolvem a requalificação da oferta de bens culturais e a cooperação técnica, disponibilizando-as para a comunidade acadêmico-científica, para gestores municipais e empresariais.

Palavras clave: Turismo e Gestão. Cooperação Técnica. Patrimônio. Montevidéu. 


\section{Introducción}

Actualmente las ciudades representan $80 \%$ de la población mundial, imponiendo nuevas reglas de convivencia y nuevos acuerdos de producción, así como los nuevos conceptos de organización y desarrollo del espacio cultural de la ciudad y su relación con el factor económico y la calidad de vida. La evolución y transformación de los espacios urbanos a través del tiempo y la urgencia de entienderlos como los principales entornos culturales para la vida de la población con calidad y oportunidades, así como la gestión para el desarrollo y el uso adecuado de la propiedad, se encuentran entre las motivaciones de esta investigación.

El estudio incluye que las ciudades es un terreno fértil para el turismo, ya que contiene la historia y la cultura de su pueblo, de su conocimiento cotidiano y prácticas inscritos en la vida cotidiana de cada uno y la formación de sus activos. Aún así, relaciona objetivos políticos, económicos, socio-culturales y ambientales de una red de interrelaciones relativas a la sociedad, ya que están directamente vinculados a las actividades realizadas por sus ciudadanos.

Debido a las ciudades seren destinos y espacios propicios para el turismo, constituyen virtuoso proceso cíclico de la cultura, en cuyo entornos culturales, económicos, políticos, sociales y tecnológicos se generan condiciones de bienestar, calidad de vida y de hospitalidad, que son condiciones para el desarrollo socioeconómico con necesidad de gestión. Turismo en estos entornos urbanos, ofrece ejemplos de experiencias y de acciones exitosas que constituyen el conocimiento para ser socializados con otras ciudades opotencialmente destinos culturales turísticos.

En este sentido, la estrategia de proporcionar conocimientos en ciudades turísticas es la cooperación técnica, orientado al desarrollo socio-económico a través de la mejora y del uso adecuado del patrimonio local. La cooperación técnica, especialmente a nivel internacional, entre países, se lleva a cabo sobre la base de la producción y de la aplicación de conocimientos a través de materiales de enseñanza, consultoría especializada, flujo de información, intercambios profesionales y tecnológicos y capacidades interrelacionadas.

A partir del marco común para la comprensión conceptual del patrimonio y el turismo cultural con el marco teórico de la cooperación técnica, la ciudad de Montevideo es el contexto del estudio en este artículo. Sus ventajas competitivas como el capital del Mercosur, se intensifican con la creación del conglomerado de turismo cultural en 2008. Factores como la prestación de servicios y atracciones turísticas, el patrimonio histórico y cultural y la creación del cluster de turismo cultural como estrategia de aumento de su desarrollo socioeconómico se analizan en este estudio.

\section{Metodología}

Fue adoptado el método descriptivo-explicativo y el estudio de caso con un enfoque cualitativo. Los procedimientos de campo incluyen el análisis de contenido del proyecto del cluster del turismo cultural de Montevideo, archivos de informes de actividad de investigación de las organizaciones públicas y privadas de la capital y de los actores turísticos de Uruguay y la observación directa del entorno turístico creativo de la ciudad. 
El enfoque metodológico consiste en la identificación de: 1) los principales aspectos históricos, culturais y turísticos de Montevideo; 2) la cooperación técnica entre la capital uruguaya y otras ciudades, instituciones o países para la planificación y la gestión y el desarrollo del proyecto del cluster de turismo cultural; 3) el potencial y las oportunidades de cooperación técnica en materia de turismo, en la actualización de turismo en Montevideo.

\section{Turismo, Patrimonio y la Cultura de la Creatividad}

La Cultura es "un mecanismo que integra el individuo a un conjunto de prácticas, costumbres, representaciones constitutivos de su entorno social" (MAFFESOLI, 1996, p. 326). Es inherente al ser humano, está presente desde el nacimiento y la voluntad la mejora de toda la vida, por lo que no es estática. Para Morin (1998) una cultura produce y reproduce modos de conocimiento entre los hombres y fortalece creencias. Burns (2002, p.75) explica la cultura como un conjunto complejo de elementos que comprenden "los conocimientos adquiridos, las creencias, el arte, la ley moral, la ley y las costumbres y otras capacidades y hábitos adquiridos por el hombre”.

En este contexto, se consideran dos tipos de cultura: la cultura material - se refiere a los aspectos físicos de la vida cotidiana, hogar, lugar de trabajo, ropa, comida, transporte y materias primas; y la cultura inmaterial - a entender las maneras de utilizar los objetos, costumbres, creencias, filosofías, los gobiernos y los estándares de comunicación (BURNS, 2002). Se basa en el vínculo entre el pasado y el presente, el contacto de los que viven con el legado y las tradiciones que fueron influenciados por la dinámica del tiempo, sino que permanecen con las formas de expresión que revela el conocimiento y hacer de cada comunidad (MAFFESOLI, 1996). De la cultura de la gente se puede identificar su patrimonio cultural. El patrimonio cultural inmaterial se define por la UNESCO como la parte de renta variable de la manifestación de las personas. Incluye las tradiciones, las costumbres, el folclore, los idiomas de las partes, entre otros.

El patrimonio cultural inmaterial es transmitido de generación en generación y recreado constantemente por las comunidades debido a su entorno, la interacción con la naturaleza o la historia, crea una continuidad que identifica a cierta población (ASHTON, 2006). Son manifestaciones que respeten la formación de la sociedad y de entender los conocimientos y acciones de un población. El patrimonio es un legado, una herencia y debe preservarse mediante la adopción de una política cultural que se ocupa de la memoria, la recuperación y la preservacion de los bienes y sus referencias culturales.

El turismo cultural, a su vez, abre perspectivas de recuperación y revitalización de la propiedad, el renacimiento de las tradiciones, el redescubrimiento de los bienes culturales a menudo amortiguada por la condición de la sociedad posmoderna (COSTA, 2009). El turismo cultural está motivado por la búsqueda de información, nuevos conocimientos, la interacción con otras personas, comunidades y lugares, las costumbres, la tradición y la identidad cultural (SEGALA, 2003). Las cosas del conocimiento del hombre son la oferta cultural, por lo que el turismo cultural tiene como objetivo conocer la bienes inmateriales y materiales producidos por el hombre, conocer algún aspecto de la cultura humana, que busca satisfacer el deseo de emociones artísticas e informação culturales (BARRETTO, 2003).

Para Barretto (2006), el turismo cultural tiene como objetivo destacar los logros humanos: la experiencia, la información obtenida en las experiencias de los turistas, la vinculación de la cultura de la comunidad local con el viajero/turista. Hay una interacción entre el lugar visitado y los que lo visitan. Se centra en la realización de los viajes motivados por el interés en la oferta cultural del destino. 
Por lo tanto, Barretto (2006), señala que la práctica del turismo cultural tiene como objetivo enriquecer la personalidad humana a través de información, conocimientos y contactos que vienen de las experiencias del viaje, consolidado a través del contacto con el patrimonio. Es la prueba más clara de la existencia de vínculos con el pasado antes de la necesidad social del hombre a experimentar una sensación de continuidad en el tiempo y la identificación con una cierta tradición (ASHTON, 2006).

Identifica así la cultura de un pueblo, a través de la continuación y la preservación del patrimonio de la empresa, a través del desarrollo de políticas públicas para su gestion. Los elementos culturales del pueblo son responsables por la atracción de los flujos turísticos y constituye uno de los ejes del turismo cultural, así como el valor universal de intercambio humano y la interacción entre las culturas, costumbres y hábitos diferentes (SEGALA, 2003).

El turismo creativo a su vez debe proporcionar una experiencia de viaje más auténtica y significativa de la vida cotidiana, de la vida de cada día de la comunidad receptora. Además, para proporcionar el desarrollo y aprendizaje del personal, es necessaria la participación activa del individuo en la experiencia turística, con la comunidad de acogida. Turismo creativo promueve el vivir como el sitio, que es parte de la vida local y crea algo juntos (co-creación), un diálogo que desarrolla la capacidad de los locales, lo que es intangible e inimitable (RICHARDS, 2013).

Como afirma Richards (2013), el turismo creativo se basa en el consumo de experiencia de creación que implica una participación activa y la participación de los consumidores en su producción. Se trata de un cambio de valores en la producción y el consumo del turismo, la experiencia hegemónica de una vida cultural local. Por lo tanto, la cultura cotidiana del lugar, lo que es la propia comunidad es el combustible para el turismo creativo. Entre los ejemplos de turismo creativo citados por Richards (2013) es el tango en la calle Buenos Aires, la integración de las ideas de Gaudí en Barcelona, la esencia relacionada con la comida en Tailandia, moda y el diseño en Milán, por último, el conocimiento colectivo que conecta a las personas, generando más ambiente para la creatividad que puede reflejar en más calidad de vida y más experiencia.

Para Reis y Kageyama (2011, p. 51), el turismo merece una mención especial, ya que "la presencia de turistas aumenta la autoestima en relación con el medio ambiente de la ciudad y por lo tanto buscamos conocer y calificarlo para nosotros los residentes y visitantes "lo que genera también un aumento de las oportunidades de empleo e ingresos, por lo que los beneficios para la comunidad de acogida.

Para Duarte (2011), el consumo revela lo que las personas son, valores, ideologías políticas o lazos emocionales. Por lo tanto, subrayamos el valor añadido para el consumidor. La creatividad de intangible genera valor adicional, por lo que el consumidor como protagonista de sus decisiones de consumo y de la identidad cultural que le da un carácter único a los bienes y servicios, es el principal factor decisivo.

En este contexto, la producción y el consumo de bienes y servicios de interés para el turismo se dan en el espacio social modificado y comprenden el conjunto de elementos materiales transformados por las prácticas económicas, apropiado para las políticas y prácticas establecidas en el significado de las prácticas sociales, culturales e ideológicas.

Como afirma Reis (2012), la producción y consumo de bienes y servicios de interés turístico promover el desarrollo basado en la cultura y no puede cumplir con cualquier modelo importado. Debe producir para los residentes y visitantes en un estado permanente de innovación, repensar en vivo culturales ciudad, proponiendo nuevos usos del patrimonio y el diálogo con sus habitantes. 
Las ciudades queinvierten en las prácticas de gestión queimplican su capital en busca de la competitividad económica y la calidad de la vida urbana, vienen con una ventaja competitiva, la producción de productos y servicios con nuevas propuestas y ofrecer nuevas vocaciones a los destinos ciudades. Se enfrentan a nuevos retos, incluida la formación de mano de obra calificada, la participación de la sociedad civil en el proceso de producción, el disfrute de los bienes y el consumo de estos bienes.

\section{Cooperación técnica}

La cooperación técnica internacional es una de las modalidades de cooperación para el desarrollo, junto con la cooperación financiera, la ayuda humanitaria, la cooperación científica y tecnológica y la ayuda alimentaria. La cooperación técnica horizontal contextualiza en las relaciones entre los países en desarrollo, y la cooperación técnica vertical, incluye las relaciones entre los países desarrollados y en desarrollo.

El concepto y la metodología de la cooperación técnica fueron establecidos por las Naciones Unidas con el fin de promover el desarrollo socio-económico. Su ámbito de aplicación es el fortalecimiento de las instituciones para la transferencia de conocimientos y tecnologia. Para la Agencia Brasileña de Cooperación ( $\mathrm{ABC}$ ) del Ministerio de Relaciones Exteriores de Brasil, el objetivo de la cooperación técnica internacional (CTI) es contribuir al desarrollo socioeconómico.

La cooperación horizontal fortalece las relaciones y los vínculos políticos y económicos más estrechos de los países. La cooperación recibida del extranjero es la interiorización de la experiencia técnica proporcionada por las organizaciones internacionales (cooperación multilateral) y los países desarrollados (cooperación bilateral), en la vista de acelerar el proceso de desarrollo nacional.

El buen gobierno, la propiedad local, la asociación y la sostenibilidad se han convertido en las directrices fundamentales de la nueva concepción de la cooperación técnica internacional. En este sentido, aunque con cierto sesgo de la verticalidad y la asimetría, se destacó la cooperación técnica entre los países en desarrollo (CTPD) en el contexto de horizontales relaciones Sur-Sur (BERG, 1993; LOPES, 2005).

Para Souza (2011, p. 76), "hay relativamente pocos estudios empíricos disponibles sobre la cooperación para el desarrollo Sur-Sur". No existen datos fiables sobre los orígenes y destinos de los recursos; o tenemos marco conceptual mínimamente aceptamos que nos permiten establecer criterios para distinguir la cooperación (o ayuda) para el desarrollo de otros tipos de cooperación, como los militares, y los préstamos, las exportaciones de servicios o la inversión extranjera directa. (SOUZA, 2011).

Para el desarrollo socio-económico, la libertad de elección y la participación ciudadana en los procesos de decisãos destinos sociales e institucionales son determinantes. (SEN, 2000). La participación popular y la democratización del acceso a las oportunidades y los beneficios del crecimiento económico para permitir que por medio de la educación y la formación para la acción, basado en una idea de los desafíos de la transformación de la realidad. (BARQUERO, 2001).

Además de los aspectos educativos, formadores, desarrollo humanístico y socio-cultural, la defensa de la independencia de las sociedades subdesarrolladas mediante la industrialización y exportación es el marco teórico de diversos autores clásicos de la economía. (FURTADO, 2005). Para el economista argentino Raúl Prebisch (1949), autor de la teoría de la CEPAL y uno de los creadores de la Comisión Económica para América Latina y el Caribe (CEPAL), el deterioro de los términos de intercambio, es decir, la pérdida de 
poder aquisivo los países desarrollo debido a la devaluación de sus productos agrexportáveis relativa a las importaciones encareciemnto condenan la tercera explotación mundial y el retroceso.

Por esta razón, es fundamental la cooperación técnica entre las ciudades creativas en varios países. Las ciudades creativas cuyo turismo es la actividad económica representante, tanto en continentes desarrollados, como en América Latina, pueden cooperar técnicamente para el desarrollo turístico, basado en diversas teorías económicas, como la teoría de la CEPAL.

\section{Caso Montevideo: la historia y el turismo}

Montevideo es la capital y ciudad más grande de la República Oriental del Uruguay, con una población de 1.319.108 habitantes ${ }^{1}$, es la sede administrativa del Mercosur y de la Asociación Latinoamericana de Integración (ALADI). Se destaca por un urbanismo abierto, con amplios espacios verdes, y diversos atractivos históricos, patrimoniales y culturales. Desde el punto de vista turístico, es el principal destino de visitantes al país según datos del Ministerio de Turismo y Deporte².

\section{Grafico 1 - Visitantes ingresados a Uruguay Destino principal del viaje: Montevideo 2002-11}

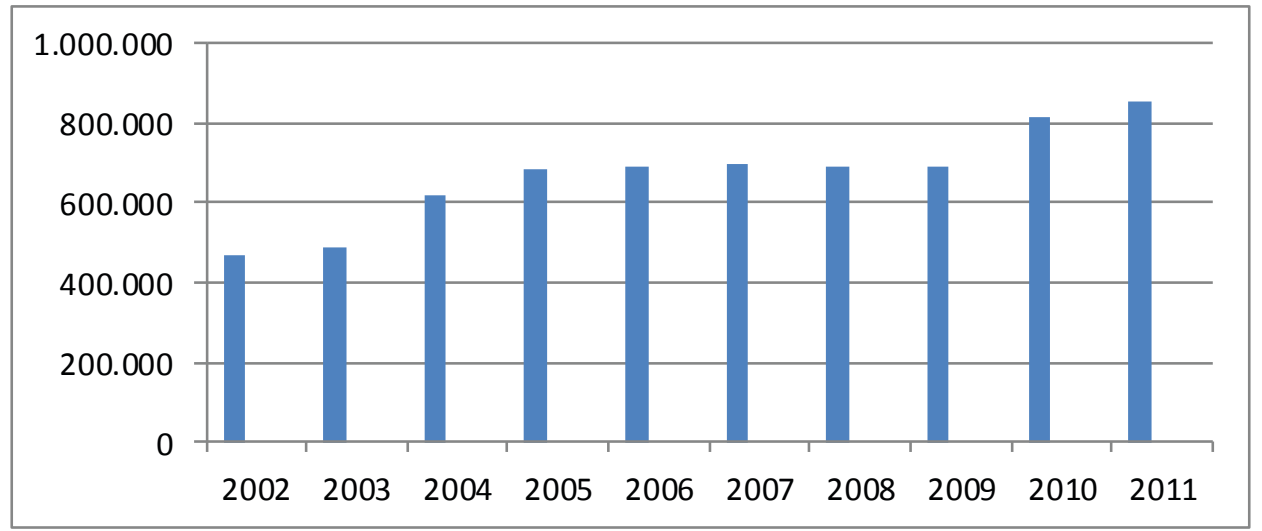

Fuente: MINTUR - Uruguay, Anuario 2012

Se puede considerar que el turismo en Uruguay tiene su origen en la ciudad de Montevideo, de esta manera en las primeras décadas del siglo XX, los proyectos turísticos se concentraron mayoritariamente en esta ciudad. $\mathrm{Y}$ así fue concebido desde el Estado - tanto nacional y municipal - siendo el Presidente José Batlle y Ordóñez $z^{3}$ quien concibió el fomento del turismo como industria en la cual confiaba en sus posibilidades y promovía la inversión. De esta manera, se la empezó a conocer como Montevideo ciudadbalneario.

En 1930, Uruguay conmemoraba el centenariodel inicio de su vida independiente y ya la ciudad capital había adquirido una fisonomía moderna. La celebración del primer campeonato mundial de fútbol en ese año proyectaba al país a nivel internacional.

Así el predominio de Montevideo en relación al turismo era indiscutido, los $22 \mathrm{~km}$ decosta a los cuales los gobiernos departamentales, o el central, le habían aportado unabuena infraestructura en materia de

1 Datoscensales 2011 en www.ine.gub.uy

2 Enadelante MINTUR

3 Fue presidente de la República en dos periodos 1903-07 y 1911-15 
calles pavimentadas, plazas, playas y parques así como los majestuosos hoteles como el Parque Hotel (1909) o el hotel Carrasco (1921), ambos con casinos, aumentaron los atractivos y hacían que se la catalogara como la capital del verano.

Si uno se pregunta ¿qué ofrecía la ciudad, cuáles eran los atractivos y cuáles los lugares de recreación en la primera mitad del siglo XX? Las respuestas se pueden encontrar en un artículo denominado Montevideo, ciudad de turismo de 1953 escrito por Alfredo M. Ferreiro quien sostenía que Montevideo tiene algo más que la playa; poseía una gama de posibilidades para los residentes ya fuese nacional o turista.

Desde la década de los treinta, la revista Turismo en el Uruguay, órgano oficial de la Comisión Nacional de Turismo publicaba una agenda de eventos compuesta por la más variada oferta pero donde el principal énfasis era puesto en el carnaval en sus diferentes modalidades, los espectáculos de fuegos artificiales y los del teatro de verano, las fiestas sociales, la doma de potros o criollas y las carreras de caballos. A su vez, los cafés, clubs y hoteles animaban la noche montevideana ofreciendo la oportunidad de bailar tango, ritmo que se consideraba de aceptación rioplatense. Como ha sostenido Da Cunha et al. (2012).

El carnaval era - y sigue siendo - uno de los principales atractivos que tenia Montevideo. Este es sin duda uno de los componentes de la identidad uruguaya ya que surge en toda la ciudad, en cada barrio, y además atraviesa todas las clases sociales. "Todo lo cual permite que se mantenga como uno de los rasgos distintivos de la imagen de Montevideo en su condición de ciudad de turismo, y a la vez, es parte de la imagen en lo que hace a su permanencia y renovación" (DA CUNHA et al., 2012, p.127).

Montevideo representaba a la perfección la idea de turismo dada su centralidad que se basaba en su conexión con otras zonas del país y de la región -dada su situación intermedia entre Buenos Aires y San Pablo - lo cual era facilitado por la concentración de vías de comunicación y de transporte.

Entonces, partiendo de esa centralidad, a mediados de la década del treinta y siguientes, los balnearios se extienden perladamente desde Montevideo. Este hacia la frontera con Brasil y van adquiriendo predominio el turismo de sol y playa, que se simboliza en la paradigmática Punta del Este. Es en este contexto, la imagende Montevideo comienza a posicionarse ahora más referida al turismo cultural y al urbano donde podrán en valor sus tradicionales recursos turísticos.

Esto se mantiene en la actualidad entonces es justo pensar ¿cómo se ha dado este proceso importante para el país? ¿Cómo comienza a conceptualizarse la idea de Uruguay país turístico? Lo que implicará poner en valor nuevas regiones del territorio al turismo.

Luego de la institucionalización del turismo a partir de la labor desarrollada por la CNdTfundamentalmente en las décadas del 1930 y 1940 - se puede observar que en las dos décadas siguientes, las políticas turísticas se desaceleraron dado el contexto del país y de la región. En este período, se debe destacar el impulso dado a la Comisión de Inversiones y Desarrollo Económico (CIDE) que en 1962 tuvo la función de evaluar la situación del país que posibilitara acceder a una mejor información para diseñar planes de desarrollo y dentro de este, también el turístico.

El informe de la CIDE señala dos componentes claros en la importancia del turismo para el país, en primer lugar como actividad económica sobre la cual expresa que "[...] es un fenómeno de origen reciente que dinamiza la actividad de otros sectores y en cuanto es externa aporta divisas al país." (CIDE, 1965, p. 1). En segundo término como actividad social pues "[...] constituye un instrumento de intercambio cultural que favorece el mejor conocimiento de los distintos grupos humanos." (CIDE, 1965, p. 1) Otro elemento que es sumamente importante de este informe es que ya plantea como aspiración a lograr "[...] la 
coordinación y complementación de los instrumentos a utilizar en los sectores público y privado." (CIDE, 1965, p. 11) Esto refiere a lo planteado sobre las políticas turísticas y como de la articulación entre ambos sectores se plasmaran los objetivos esenciales para el desarrollo turístico.

Esto provocará a partir de la década del 1970 que se realicen diversos estudios mediante el sistema de consultorías que buscaban lograr una estrategia de desarrollo. Así por ejemplo, en 1972 se presentó el Informe preparado por Clemente-Smith Inc. el cual marca un rumbo con respecto al futuro de Montevideo. Este informe parte de las prioridades del Gobierno en materia turística que eran: 1) La costa Norte del Atlántico entre Castillos y Chuy; 2) Montevideo y Punta del Este; 3) Colonia y 4) La zona de los baños termales.

Sin embargo, los consultores entienden que las prioridades deben ser revisadas y centrar las futuras estrategias en la prioridad $\mathrm{N}^{\circ} 2$ dado que se consideraba que: “[...] tiene comodidades y medios de alojamiento en el área de Prioridad $\mathrm{N}^{\circ} 2$ (Montevideo-Punta del Este) y estas ventajas no se usan debidamente. Por lo tanto, a menos que el Uruguay pueda atraer una gran cantidad de financiación excepcional [...] sería mejor promover la prioridad $\mathrm{N}^{\circ} 2$ a la posición $\mathrm{N}^{\circ} 1$ [...]" (CLEMENT-SMITH INC, 1972, p.120-121).

Esto es ratificado posteriormente en 1978 por un informe realizado por CICATUR -OEA el cual sostiene que: "En el análisis de la oferta se identificaron tres zonas, Colonia, Termas y Montevideo, cuyo potencial turístico no estaba evaluado y sobre los que por lo tanto existían expectativas no fundamentadas en el conocimiento sistematizado" (CICATUR-OEA, 1978, p. 309).

Estos antecedentes son fundamentales para las políticas y/o acciones llevadas a cabo en las décadas siguientes por la Intendencia Departamental así como por el Ministerio de Turismo - creado en 1986 - y que aportará a los criterios de actualidad que tiene la ciudad dentro del contexto nacional.

Si bien el gobierno departamental de Montevideo desde comienzos del siglo XX tiene una activa y protagónica participación en las definiciones turísticas de la ciudad a comienzos del siglo XXI aparecen nuevas figuras organizativas que acompasan los nuevos tiempos: la Corporación Turística de Montevideo y el Conglomerado Turístico de Montevideo (2013).

De acuerdo a lo sostenido por Claudio Quintana 4 : En la década de los noventa y principios del 2000 a raíz de la crisis de la desindustrialización de las economías urbanas y del fracaso de las políticas neoliberales aplicadas en nuestro país, en Montevideo se volvió la mirada al turismo como fórmula de regeneración socioeconómica urbana. El desarrollo urbano y el turismo como a principios de siglo, nuevamente eran complementarios, pero ahora en otro ámbito territorial de Montevideo. Se generó un nuevo impulso de la política pública municipal creando la Corporación Turística de Montevideo (primer antecedente de institucionalidad pública- privada en Montevideo y de planificación turística con criterios científicos), se realizaron inversiones públicas en equipamientos e infraestructuras en la Ciudad Vieja y la activación patrimonial del Tango y el Candombe que concluyeron en el 2009 con la declaración de Patrimonio Cultural Inmaterial de la Humanidad en una estrategia compartida entre Montevideo y Buenos Aires ante la UNESCO (QUINTANA, 2014).

En cuanto a la Corporación Turística de Montevideo, ésta fue creada en 2001 bajo la forma de una comisión asesora municipal y sus funciones básicas eran las siguientes: a) elaborar la política turística de Montevideo; b) promover el desarrollo de la oferta turística, recreativa y cultural; c) promover iniciativas legales y administrativas para el desarrollo turístico; d) promover y organizar campañas de promoción y difusión; y e) coordinar actividades y promover el intercambio entre el sector público y el sector privado.

4 Licenciado en Turismo, exDirector de Turismo de lalntendencia departamental de Paysandú y de lalntendencia departamental de Montevideo. 
Se le otorga una estructura organizacional con un órgano ejecutivo y un plenario, contemplando la participación intersectorial al quedar incorporadas la Intendencia Municipal, el Ministerio de Turismo, los actores privados vinculados directa o indirectamente a la actividad y la Universidad de la República. Es decir, el plenario estaba integrado por organizaciones públicas y privadas vinculadas estrechamente con la producción turística de la ciudad y el departamento. Éste, a su vez, organizaba distintos grupos de trabajo temáticos, donde participan tanto organizaciones como personas, integrados para elaborar y ejecutar los proyectos.

La Corporación Turística de Montevideo buscó favorecer el desarrollo del sistema mediante la planificación y promoviendo la asociación y la cooperación entre los distintos actores. A vía de ejemplo, en su plan de 2003 tuvo como fin el desarrollo turístico de Montevideo, mediante una explotación integral y sustentable de los recursos provenientes del ambiente natural, el patrimonio cultural y el capital social de Montevideo.

El ex Director de Turismo de la Intendencia de Montevideo, Sr. Fernando González manifestó en una entrevista de prensa que la Corporación Turística de Montevideo: Evolucionó buscando su formato ideal hasta lo que hoy se consolidó, el Conglomerado de Turismo de Montevideo, que se amparó desde un principio en un programa financiado por el BID [Banco Interamericano de Desarrollo] pero ya tiene vida propia como asociación civil de privados con participación del Ministerio y la Intendencia ${ }^{5}$.

Esto es ratificado por el actual Director de Turismo de la comuna, Sr. Rafael Unanian, quien en un desayuno de trabajo del Conglomerado en 2013 manifestó la importancia del espíritu de integración público-privada que ha promovido el gobierno nacional y el gobierno departamental desde la creación del Conglomerado de Turismo de Montevideo. Remarcó la continuidad de esta política de articulación que ya lleva más de cinco años y que tiene sus antecedentes en la Corporación Turística de Montevideo, creada en 2001 en el marco de la Intendencia.

El Conglomerado de Turismo surge en 2008, en base a los diagnóstico realizados mediante el Programa de Competitividad de Conglomerados y Cadenas productivas Préstamo 1763 / OC-UR.

De este modo se conforma una nueva alianza estratégica de carácter público-privada, en la cual se define un plan estratégico para Montevideo y se plantean líneas de acción para la ejecución del mismo. Se define como. "es un espacio de encuentro, coordinación, planificación y ejecución de acciones estratégicas, entre actores públicos y privados organizados, con el objetivo de mejorar la competitividad turística de la ciudad"6.

De esta manera, los ejes principales sobre los que trabaja son la planificación y la articulación de todo lo que conlleva ese proceso de coordinación entre los diferentes sectores involucrados de forma directa o indirecta en la actividad turística.

La visión que se planteó en el diagnóstico original condice con ello: "Ser el destino de referencia del Río de la Plata para el turismo de negocios y short-breaks, a partir de la puesta en valor de la oferta cultural y de ocio del destino y de sus valores en alza como la seguridad y la hospitalidad de la gente; ofreciendo un portafolio de turismo diversificado para satisfacer al mercadode demanda regional e internacional, potenciado a partir de los visitantes uruguayos residentes en el exterior"7.

\footnotetext{
5 Entrevista realizada por La Diariael 11.5.2010 en http://ladiaria.com.uy/articulo/2010/5/montevideo-turistico/

6 Dossier institucional del Conglomerado

7 Turismo en Montevideo - Plan de Refuerzo de la Competitividad (PRC)
} 
La actividad del conglomerado está enfocada en el fortalecimiento continuo de cuatro pilares, estos son: la planificación estratégica, el cofinanciamiento, la coordinación técnica y la articulación de todos los sectores. Se trabaja en función de un plan estratégico presentado ante públicos y privados en 2009 denominado "Plan de refuerzo de la competitividad", del Conglomerado de Turismo de Montevideo. El cual sobre la base de una metodología de análisis estratégico y participativo pretende el desarrollo de un proceso de cambio y dinamización de conglomerados productivos.

El ex Presidente del Conglomerado, Cr. Federico Celsi cuando se lo consultó sobre cómo surgió la idea de este emprendimiento afirma que se presentaron a una convocatoria de Dirección de Proyectos de Desarrollo del Estado (DIPRODE), quien tuvo la iniciativa fue la Dirección de Turismo de la Intendencia de Montevideo y que existían distintas propuestas a ser financiadas pero finalmente se obtuvo el financiamiento de unos U\$S 500.000 que se pueden destinar a actividades turísticas. A su entender esto representa algo más que el dinero consideró que fue “(...) la oportunidad histórica que tiene Montevideo de trabajar todos juntos, los empresarios y las estructuras públicas, para mirar a Uruguay a largo plazo y no constantemente pensar en medidas cortoplacistas." En la misma entrevista el ex Coordinador del Conglomerado, Oscar Iroldi, sostiene que en los talleres realizados por la consultora: “(...) todos definieron a Montevideo con un ítem muy valorado que es la gran oferta cultural y de ocio que tiene la ciudad, que nos posiciona dentro de las grandes capitales que ofertan esto en América Latina y en el mundo. Ahí hay una línea estratégica."

En cuanto a la percepción de lo realizado por parte del sector público, la Intendenta de Montevideo, Prof. Ana Olivera, expresó que se "está culminando con un año excepcional marcado por la celebración de Montevideo Capital Iberoamericana de la Cultura 2013. Esta designación posicionó a Montevideo de otra manera frente al mundo"10. También señaló la importancia de la reinauguración del Hotel Carrasco y del Mercado Agrícola, así como de la presencia de las grandes cadenas hoteleras internacionales que se están instalando en la ciudad.

Quadro 1 - La estructura actual del Conglomerado es la siguiente:

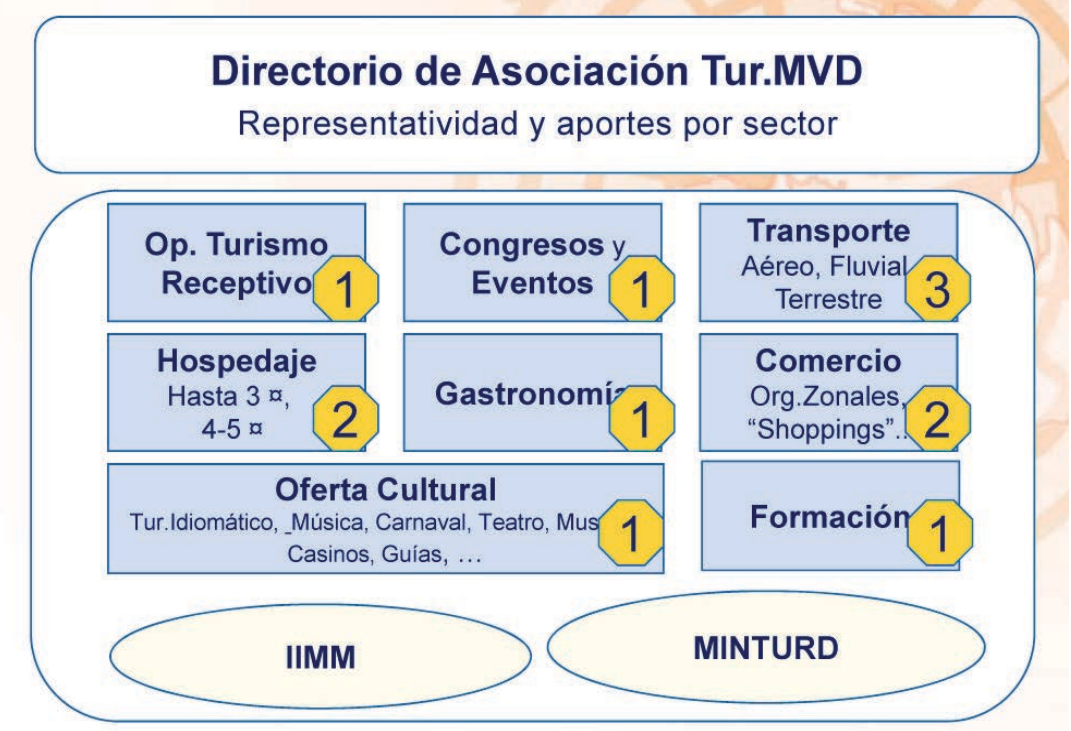

Fuente: Turismo en Montevideo - Plan de Refuerzo de la Competitividad (PRC)

8 Fuente: Turismo en Montevideo - Plan de Refuerzo de la Competitividad (PRC)
9 lbíd

10 En http://www.uruguaynatural.com/ 
Como se desprende del organigrama la preocupación por el turismo cultural se manifiesta en la ponderación en la representatividad del sector. Y de alguna manera esto define una posición clara en cuanto a la presentación de Montevideo como un destino de turismo cultural. Esto tiene varios fundamentos que han sido analizados desde ópticas transversales. Un informe elaborado por el Sector Cultura de la UNESCO en 2008 denominado "Consolidando el Uruguay Cultural" parte de algunas premisas iníciales como ser: existe en Uruguay una gran potencialidad para la implementación de políticas

Centradas en el desarrollo cultural y la implementación de políticas integradas de desarrollo cultural es potencialmente positiva en tanto permita el aprovechamiento y la creación de sinergias entre los agentes públicos y privados involucrados.

En este sentido el documento sostiene que : "Posicionar al Uruguay Cultural, es contribuir a largo plazo con las nuevas identidades asociadas con la naturaleza, con el desarrollo del turismo cultural interno y su inclusión en el contexto regional, con marca de identidad propia, ofreciendo calidad de sus servicios en los itinerarios turísticos de patrimonio cultural de la región MERCOSUR." (UNESCO, 2008, p. 4).

Este informe señala que existen dos componentes culturales que se encuentran bien posicionados, estos son: el sector audiovisual - queha crecido en términos de su producción y de la proyección nacional e internacional - yel carnaval tiene gran potencialidad en términos de desarrollo de iniciativas que incluyan su explotación turística.

Atendiendo que lo cultural atraviesa la vida social, es fundamentalque la transversalidad sea pensada por los agentes responsables de las políticas públicas desde la formulación, la toma de decisiones y la ejecución. En este sentido, el Conglomerado ha tomado en consideración estos aspectos y su preocupación así como diseño de acciones para posicionar a Montevideo como un destino de turismo cultural ha sido considerable. Esto ya estaba de manifiesto en el diagnóstico realizado y en cuanto a los recursos culturales de los que dispone la ciudad se da cuenta en el mismo.

Si se analiza cual es el trabajo que viene desarrollando el conglomerado sobre turismo cultural se encuentran los siguientes productos en marcha de acuerdo al dossier institucional: Descubrí Montevideo Gastronómico, Agenda Cultural Anual, Actualización del Plan Estratégico de Turismo en Montevideo 2016, Montevideo + Museos y Pase de Museos.

En tal sentido y a modo de balance de las acciones realizadas por el Conglomerado en 2013, el ex Presidente del mismo, Sr. Jorge Erramouspe, señaló que entre lo efectuado se encuentran la actualización del plan estratégico de turismo de la ciudad, el que se implementará entre 2014 y 2016. En materia de promoción mencionó el lanzamiento de la campaña Montevideo Te Beneficia, la presencia del destino en ferias internacionales, la organización de viajes de prensa de medios internacionales, y la realización de dos nuevos productos turísticos: Descubrí Montevideo Gastronómico y Montevideo + Museos. Entre los principales desafíos para el 2014 puso un énfasis especial en la concreción del bureau de congresos y convenciones que fomente el crecimiento del turismo de reuniones.

\section{Reflexiones finales}

Montevideo como destino turistico tiene una larga trayectoria de mas de un siglo, en la cual la ciudad se ha ido adecuando a los cambios sociales y a las practicas turisticas, comenzando como ciudad balnearia y recostada a su larga costa hasta la epoca actual como un destino cutlural y de negocios de signficacion 
en el Cono Sur. En este largo proceso siempre ha tenido un papel prepoderrante el gobierno de la ciudad (Intedencia departamental) y el gobierno central, quienes han destinado recursos para los planes de fomento turistico asi como para la adecuacion de la infraestructura urbana.

Ha sido objeto de analisis para el desarrollo de estrategias por parte de distitnos organismos desde la CNdT, pasando por consultoras intenacionales, la OEA y la UNESCO.

A partir del siglo XXI ha comenzado un interesante proceso de colaboracon publico/privada que tiene hoy su expresion en el Conglomerado Turistico de Montevideo quien busca mejorar la imagen y el posicionamiento de la ciudad ante los cambios que se han producido en los conusmidores turisticos.

Tomando en consideracion la caracterizacion realizada anteriormente sobre turismo creativo se puede afirmar que Montevideo está en condiciones de llevar adelante este tipo de propuestas ya que los turistas conusmen cultura en un sentido amplio en el destino, a traves del Conglomerado se propician nuevos emprendimientos y productos culturales, se pone en valor la identidad de la ciudad desde una perspectiva transveral, se genera permanentemente el consumo de bienes culturales dentro de una amplia oferta y se intensifican el intercambio cultural asi como los modos y estilos de vida entre los residentes y los turistas .

Entonces si bien actualmente Montevideo centra su oferta en el turismo cutlural y entendiendo que el turismo creativo es fundamentalmente un turismo de tercera generacion donde se percibe mayor flexibilidad en las formas tradicionales de turimo cultural se debe valorar en un periodo futuro como usa estas herramientas el Conglomerado, cuestion que solo podra ser evaluada en el mediano plazo.

\section{Referências}

AGÊNCIA BRASILEIRA DE COOPERAÇÃO (ABC). Disponível em: 〈www.abc.gov.br〉. Acesso em: 30 out. 2014 .

ASHTON, M. S. G. Turismo: a mutação do cotidiano. In: BARRETTO, M. (Org.) Turismo, cultura e sociedade. Caxias do Sul, RS: EDUCS, 2006, p.11-24.

BARRETTO, M. Turismo e legado cultural. Campinas, SP: Papirus, 2003.

. Turismo, cultura e sociedade. Caxias do Sul, RS: EDUCS, 2006.

BARQUERO, A. V. Desenvolvimento endógeno em tempos de globalização. Porto Alegre: UFRGS Editora, 2001.

BERG, E. J. Rethinking technical cooperation: reforms for capacity building in Africa. New York: UNPD/ DAI, 1993.

BURNS, P. M. Turismo e antropologia: uma introdução. São Paulo, SP: Chronos, 2002.

CICATUR, OEA. Desarrollo turístico del Uruguay - Lineamientos para una estrategia Desarrollo Turístico. Estrategia: Serie de Informes y Estudios, n. 25 - Tomo I -Washington DC, 1978.

CLEMENTE-SMITH INC. Siete Pasos para acelerar el turismo en el Uruguay. Washington DC, 1972. COMISSÃO ECONÔMICA PARA A AMÉRICA LATINA E O CARIBE. Disponível em: 〈www.cepal.org/pt-br〉. Acesso em: 10 out. 2014.

CONGLOMERADO DE MONTEVIDEO. Dossier Institucional.pdf, 2013. 
COSTA, F. R. Turismo e patrimônio cultural: interpretação e qualificação. São Paulo, SP: Senac/Sesc, 2009.

CUNHA, N. da. et al. Visite Uruguay: del balneario al pais turístico. Ediciones de la Banda Oriental: Montevideo, 2012.

DOSSIER DE ECONOMIA CRIATIVA. 2010. Agência Inova CultDigest, Lisboa. Disponível em: 〈http:// www.esar.edu.pt/be/ficheiros/Recursos/Economia/Dossier_Economia_Criativa.pdf $>$. Acesso em: 09 set. 2013.

DUARTE, A. Experiências de consumo. Revista Etnográfica, v. 15, n. 3, 2011.

FERREIRO, A. M. Montevideo, ciudad de turismo. Almanaque del Banco de Seguros, 1953, bse.com.uy, p.420-28, 1953. Entrada el 06/06/14.

FURTADO, C. Formação econômica do Brasil. São Paulo: Companhia Editora Nacional, 2005.

LEITE, R. P. Contra-usos da Cidade: lugares e espaço público na experiência urbana contemporânea. Campinas, SP: Unicamp; Aracaju, SE: UFS, 2007.

LOPES, C. Cooperação e desenvolvimento humano: a agenda emergente para o novo milênio. São Paulo: Unesp, 2005.

MAFFESOLI, M. A Contemplação do Mundo. Porto Alegre: Artes e Ofícios, 1996.

MINTUR. Anuario Estadístico 2012, Montevideo, 2013.

MORIN, E. $O$ Método 4. As Ideias: habitat, vida, costumes, organização. Porto Alegre: Sulina, 1998.

OFICINA REGIONAL de Ciencia de la UNESCO para América Latina y el Caribe (2008) Políticas transversales en cultura, turismo y ambiente: desafíos y oportunidades en Uruguay, Montevideo.

PLAN NACIONAL DE DESARROLLO ECONÓMICO Y SOCIAL 1965-1974 TII A Síntesis de planes económicos, Mdeo, 1965.

PROGRAMA de Competitividad de Conglomerados y Cadenas Productivas. Turismo en Montevideo Plan de Refuerzo de la Competitividad (PRC), Uruguay, 2009.

QUINTANA, Disponível em: 〈http://claudioquintana.blogspot.com/2013/04/〉 Acesso em: 27 abr. 2014 .

REIS, A. C. F.; KAGEYAMA, P. Cidades criativas: perspectivas. São Paulo: Garimpo de Soluções, 2011.

REIS, A. C. F. Cidades Criativas, Turismo Cultural e Regeneração Urbana. 2012. Disponível em: 〈http:// www.gestaocultural.org.br/pdf/Ana-Carla-Fonseca-Cidades-Criativas.pdf $>$. Acesso em: 12 mar. 2014.

RICHARDS, G. Palestra na 1aㅡ Conferência Brasileira de Turismo Criativo - Porto Alegre, RS, em 22 de outubro de 2013.

SEGALA, L. V. Gastronomia e Turismo Cultural. Revista Turismo. 2003. Disponível em: 〈http:// revistaturismo.com.br/materiasespeciais>. Acesso em: 12 mar. 2014.

SEN, A. Desenvolvimento como liberdade. São Paulo: Companhia das Letras, 2000.

SOUZA, A. M. A Cooperação para o desenvolvimento Sul-Sul: os casos do Brasil, da Índia e da China. Boletim de Economia e Política Internacional, v. 9, p. 89-99, 2011. 
UNESCO. Convenção para a Salvaguarda do Patrimônio Cultural Imaterial. 2008. Disponível em: 〈http://www.unesdoc.unesco.org/images/0013/001325/132540s.pdf〉. Acesso em: 27 abr. 2014.

URUGUAY, 2014. Disponível em: 〈www.ine.gub.uy〉 Acesso em: 27 abr. 2014.

URUGUAY, 2013. Disponível em: 〈www.uruguaynatural.com〉 Acesso em: 27 abr. 2014. 